\title{
Quality Standards versus Taxation in a Dynamic Environmental Model of a Tourism Economy
}

\author{
Rey-Maquieira, J. ${ }^{1}$, J. Lozano' ${ }^{1}$, C.M. Gómez ${ }^{2}$ \\ ${ }^{1}$ Department of Applied Economics, University of the Balearic Islands, Spain \\ ${ }^{2}$ Department of Economics and Economic History, University of Alcalá, Madrid, Spain
}

Keywords: tourism economics, tourism policy, tourism taxation, quality standards, economic modelling

\section{EXTENDED ABSTRACT}

The paper constructs a dynamic general equilibrium model for a small economy that specializes in tourism to explore the long term effects of environmental and tourism policies. The model assumes that tourists obtain satisfaction from private services provided by tourism firms, goods and services provided by the public sector and environmental quality. Satisfaction from private services depends on the quality of those services, which is represented by the amount of capital per unit of accommodation. It is also assumed that goods and services provided by the government suffer from congestion. Environmental quality, which is also valued by the residents, is modelled as a renewable resource that is depleted by tourism activity. Tourism firms are price takers in the international tourism market, in the sense that the amount of tourism services provided by the economy has no direct impact on international prices. The price, however, depends positively on the quality of the supplied tourism bundle and therefore tourism firms in the destination can charge higher or lower prices depending on the quality of private tourism services, congestion of public goods and environmental quality. In this setting, the long term effects of two alternative policy instruments are compared, namely an overnight stay tax and a quality standard that is imposed on tourism firms. Both instruments can be used as accommodation quality and environmental quality policy tools. However, their welfare consequences are different as they have different effects on the incentives to accumulate capital. Specifically, the overnight stay tax acts as an implicit tax on capital, reducing the incentives for investment and capital accumulation. From a long term perspective, the quality standard is always superior to the overnight stay tax, provided that the former does not cause any dynamic inefficiency. 


\section{INTRODUCTION}

In many cases specialization in tourism has been triggered by the abundance of natural resources. The natural environment is part of the tourism output, but tourism activity exerts a high pressure on nature (Tisdell, 2001; Davies and Cahill, 2000) which may harm the welfare of residents and even put in danger the tourism viability of the economy. Much of the environmental damage are external costs, leading to an excessive tourism expansion from the social point of view.

The increasing importance of tourism has triggered an interest in public intervention. For instance, some tourism economies strive for a change of the pattern of specialization from the mass tourism to "quality" tourism. In some cases as well, there is a demand for public intervention to correct environmental externalities generated by the tourism sector. To reach these targets several policy instruments have been used such as tourism taxes (room taxes, entry taxes and exit taxes), quality requirements imposed on the suppliers of tourism services, or the provision of public infrastructures related to the tourism activity.

In this paper we extend the dynamic general equilibrium model developed in Gómez et al. (2007) to include not only tourism taxes but also quality standards. This model is developed for a small economy specialized in tourism, where the expansion of the tourism sector is the engine of growth and which includes the relationship of dependence and impact between the tourism activity and the environment. Methodologically the model is related to the literature that extends economic growth theory to include environmental variables (Tahvonen and Kuuluvainen, 1993; Stokey, 1998; Hettich 1998, among others). In this framework, we explore the effects of some tourism-environmental policies, specifically, an overnight stay tax and a quality standard for tourism firms.

The remainder of the paper is organized as follows. Section 2 discusses the model. Section 3 compares two policy instruments in terms of the consequences for long term welfare: the overnight stay tax and a quality standard for the tourism firms. Finally, section 4 gives some concluding comments.

\section{THE MODEL}

We consider the case of a small open economy that is fully specialized in providing tourism services to foreigners.

\subsection{Tourism preferences and tourism revenues}

A visitor obtains satisfaction from three different sources:

a) Tourism services provided by the tourism firms. We consider capital relative to the capacity of accommodation as a quality index from the tourism firm, that is, $K_{i} / T_{i}$, where $K_{i}$ is the capital of the firm $i$ and $T_{i}$ is its capacity of accommodation.

b) Goods and services provided by the public sector. Some goods and services provided by the public sector are mainly for the use of those tourists that lodge in a specific area. However, the public sector also provides goods and services for the whole tourism industry. Let us call the first kind of goods and services $G_{A}$ and the second $G_{B}$. We assume that both types are subject to congestion.

c) Environmental quality of the resort. In many tourism resorts natural assets are an essential part of the tourism product and as such a key determinant of the visitors' satisfaction. We consider that environmental quality is represented by a single variable called $N$.

Given these assumptions we define a utility function that measures the satisfaction per overnight stay of a tourist that visits the resort:

$$
U_{i}^{T}=\left(\frac{K_{i}}{T_{i}}\right)^{\alpha}\left(\frac{G_{B}}{\vartheta T}\right)^{\beta}\left(\frac{G_{A i}}{\vartheta T_{i}}\right)^{\gamma} N^{\mu}
$$

where we assume that $\alpha, \beta, \gamma \in(0,1) ; \mu>0 ; \alpha+\beta+\gamma<1$

where $U^{T}{ }_{i}$ is the utility of a tourist that receives services from firm $i, G_{A i}$ are excludable goods and services that firm $i$ receives from the public sector, $T$ is the aggregate accommodation capacity of the resort and $\vartheta$ is the number of overnight stays per unit of accommodation capacity.

From (1) we can obtain the revenues that accrue to a firm given additional assumptions. Firstly, we define $P_{U}$ as the price a tourist is willing to pay for a 
unit of satisfaction obtained in the resort. Given this, the price paid for the tourism services is:

$$
P_{i}^{T}=P_{U} U_{i}^{T}
$$

Secondly, and given that the number of overnight stays in the tourism firm is $\vartheta T_{i}$, revenues of a firm $i$ are:

$$
T R_{i}=\phi T_{i}^{1-\alpha-\gamma} K_{i}^{\alpha}\left(\frac{G_{B}}{T}\right)^{\beta} G_{A i}^{\gamma} N^{\mu}
$$

where $\phi=\vartheta^{l-\beta-\gamma} P_{U}$

\section{Aggregate revenues are:}

$$
T R=\phi T^{1-\alpha-\beta-\gamma} K^{\alpha} G_{B}^{\beta} G_{A}^{\gamma} N^{\mu}
$$

\subsection{Public sector}

The public sector raises revenues using a tax levied on tourism revenues, $\tau_{T R}, 0 \leq \tau_{T R}<1$ and an overnight stay tax, $\tau_{T}, \tau_{T} \geq 0$. Public budget is always in equilibrium, that is:

$$
\tau_{T R} T R+\tau_{T} T=G_{A}+G_{B}+F
$$

where we allow for lump-sum transfers, $F$, and $\vartheta$ is normalized to one.

\subsection{Firms' behavior}

Firms maximize profits hiring capital and taking decisions over their accommodation capacity. Two conditions follow from profit maximization:

$$
\begin{aligned}
& \left(1-\tau_{T R}\right) \alpha \frac{T R}{K}=R \\
& \left(1-\tau_{T R}\right)(1-\alpha-\gamma) \frac{T R}{T}=\tau_{T}
\end{aligned}
$$

Aggregate profits are:

$$
\Pi=\left(1-\tau_{T R}\right) \gamma T R
$$

Residents are modelized as a single representative agent that maximizes the following intertemporal utility function:

$$
\omega_{0}=\int_{0}^{\infty} e^{-\rho t} \frac{\left(C_{t} N_{t}^{v}\right)^{1-\theta}}{1-\theta} d t \quad v, \rho, \theta>0
$$

where $\theta$ is the constant intertemporal elasticity of substitution, $\rho$ is the rate of time preference and $v$ is a relative weight of environmental quality on residents' preferences and $C$ is consumption.

Applying the maximum principle, the following expressions determine the behavior of residents:

$$
\begin{aligned}
& \dot{K}=r K+\Pi+F-C \\
& \frac{\dot{C}}{C}=\frac{1}{\theta}\left[r-\rho+v(1-\theta) \frac{\dot{N}}{N}\right] \\
& \lim _{t \rightarrow \infty} \lambda_{t} K_{t}=0
\end{aligned}
$$

where $r$ is net real rate of return and $\lambda$ is a co-state variable associated with capital.

\subsection{Environmental quality}

We interpret environmental quality as a renewable resource. Tourism activity has damaging effects on the environment.

We assume that environmental quality evolves over time according to the following function:

$$
\dot{N}=\varsigma(\bar{N}-N)-z T
$$

$\bar{N}$ is the maximum level of environmental quality, $\varsigma$ is the rate of recovery of the environment due to natural regeneration and $z$ measures the environmental impact associated with a unit of accommodation capacity.

\subsection{Equilibrium}

Considering $r=R-\delta$, where $\delta$ is the rate of capital depreciation we obtain: 


$$
\frac{\dot{C}}{C}=\frac{1}{\theta}\left[\alpha\left(1-\tau_{T R}\right) \frac{T R}{K}-\delta-\rho+v(1-\theta) \frac{\dot{N}}{N}\right]
$$

11)

$$
\dot{K}=T R-G_{A}-G_{B}-\delta K-C
$$

The model has a stationary state where: their $K_{i} / T_{i}$ to the legal minimum. The firm and aggregate revenue functions are respectively:

$$
\begin{gathered}
T R_{i}=\phi \kappa^{\alpha+\gamma-1} K_{i}^{1-\gamma} G_{A i}^{\gamma}\left(\frac{G_{B}}{T}\right)^{\beta} N^{\mu} \\
T R=\phi \kappa^{\alpha+\beta+\gamma-1} K^{1-\beta-\gamma} G_{A}^{\gamma} G_{B}{ }^{\beta} N^{\mu}
\end{gathered}
$$

where $\kappa$ is the quality standard.

$\dot{N}=\dot{K}=\dot{C}=\dot{G}_{A}=\dot{G}_{B}=\dot{T} R=\dot{T}=\dot{\tau}_{T}=\dot{\tau}_{T R}=\dot{F}=$ Firms' behavior is determined by the quality

$$
N=\left[\frac{1}{\phi}\left(\frac{\delta+\rho}{\alpha}\right)^{\alpha}\left(\frac{\tau_{T}}{1-\alpha-\gamma}\right)^{1-\alpha-\beta-\gamma}\left(\frac{1}{1-\tau_{T R}}\right)^{1-\beta-\gamma}\left(\frac{1}{s_{A}}\right)^{\gamma}\left(\frac{1}{s_{B}}\right)^{\beta}\right]^{\frac{1}{\mu}}
$$

13)

$$
T=\frac{\varsigma(\bar{N}-N)}{z}
$$

)

$$
T R=\frac{\tau_{T}}{\left(1-\tau_{T R}\right)(1-\alpha-\gamma)} T
$$

)

$$
K=\frac{\left(1-\tau_{T R}\right) \alpha}{\delta+\rho} T R
$$

)

$$
C=\left(1-s_{A}-s_{B}\right) T R-\delta K
$$

\section{OVERNIGHT STAY TAX AND QUALITY STANDARDS}

We compare the long-term effects of the overnight stay tax and a quality standard. We define a quality standard as a minimum of capital per unit of accommodation capacity imposed to the tourism firms by the public sector.

First of all, let us consider the behavior of the economy when there is a quality standard and no overnight stay tax. In this context firms adjust

$$
\left(1-\tau_{T R}\right)(1-\gamma) \frac{T R}{K}=R
$$

The steady state is:

$$
\begin{aligned}
N=\left[\frac{1}{\phi} \kappa^{1-\alpha-\beta-\gamma}\left(\frac{\delta+\rho}{1-\gamma}\right)^{1-\beta-\gamma}\left(\frac{1}{1-\tau_{T R}}\right)^{1-\beta-\gamma}\left(\frac{1}{s_{A}}\right)^{\gamma}\left(\frac{1}{s_{B}}\right)^{\beta}\right]^{\frac{1}{\mu}} \\
T=\frac{S(\bar{N}-N)}{z} \\
K=\kappa T \\
T R=\frac{\delta+\rho}{\left(1-\tau_{T R}\right)(1-\gamma)} K \\
C=\left(1-S_{A}-S_{B}\right) T R-\delta K
\end{aligned}
$$

Profits can be interpreted as income that accrues to an implicit fixed factor (land or know-how). When the overnight stay tax is used the tax system favors the fixed factor to the detriment of capital while the other way round happen when we opt for the quality standard. As it is well known, distortions from the tax system are minimized when the tax burden is mainly borne by the fixed factors. This suggests that the quality standard would yield a higher welfare level than the overnight stay tax. However, this is not always true since the quality standard can give place to a problem of dynamic inefficiency.

To show this, let us consider that the level of the overnight stay tax and the quality standard are such that they yield the same steady state environmental quality level. This allows us to concentrate in the differences in steady state consumption. We also assume that public expenditure relative to tourism 
revenues is set at its optimal level, that is, $s_{A}=\gamma$, $s_{B}=\beta$. For a given level of environmental quality, steady state consumption is:

$$
C=(1-\gamma-\beta) \Omega K^{\alpha /(1-\beta-\gamma)}-\delta K
$$

where

$$
\Omega=\left[\phi(\varsigma / z)^{1-\alpha-\beta-\gamma}(\bar{N}-N)^{1-\alpha-\beta-\gamma} \gamma^{\gamma} \beta^{\beta} N^{\mu}\right]^{\frac{1}{(1-\beta-\gamma)}}
$$

The expression (26) comes from (2'), (17) and the steady state relationship between accommodation capacity and environmental quality, (14).

Form (26) the level of capital that maximizes long term consumption can be worked out:

$$
K_{G R}=\left(\frac{\alpha \Omega}{\delta}\right)^{(1-\beta-\gamma) /(1-\alpha-\beta-\gamma)}
$$

where $K_{G R}$ is the golden rule level of capital. is:

With a overnight stay tax steady state capital

$$
\begin{aligned}
& K_{1}^{\tau_{T}}=\left[\frac{(1-\gamma-\beta) \alpha \Omega}{\delta+\rho}\right]^{(1-\beta-\gamma) /(1-\alpha-\beta-\gamma)} \\
& K_{2}^{\tau_{T}}=\left[\frac{(1-\gamma-\beta) \alpha \Omega}{(\alpha+\gamma)(\delta+\rho)}\right]^{(1-\beta-\gamma) /(1-\alpha-\beta-\gamma)}
\end{aligned}
$$

where $K_{1}^{\tau_{T}}$ and $K_{2}^{\tau_{T}}$ stand for the steady state level of capital when the overnight stay tax revenues are given back in lump-sum fashion and when they are used to reduce the revenue tax, respectively. As it should be expected, in the second case steady state capital is higher since the after tax returns to capital are larger. Moreover, in both cases the level of capital is below the golden rule provided that the revenue tax is positive.
Therefore, long term consumption is also higher in the second case.

When the public sector sets a quality standard, capital in the steady state is:

$$
K^{\kappa}=\left[\frac{(1-\gamma-\beta)(1-\gamma) \Omega}{\delta+\rho}\right]^{(1-\beta-\gamma) /(1-\alpha-\beta-\gamma)}
$$

It is easy to show that in this later case capital reaches a higher level in the steady state than when the overnight stay tax is used. ${ }^{1}$ However, since $\alpha<(1-$ $\gamma)$, with the quality standard the economy could end up with a level of capital above its golden rule level. Therefore, provided that the quality standard does not give place to over accumulation of capital, this instrument yields a higher long term welfare level than the overnight stay tax. Put it in a different way, a necessary (but not sufficient) condition for the overnight stay tax to be a better instrument is that under the quality standard regime the economy suffers from dynamic inefficiency.

\section{CONCLUSIONS}

We have constructed a dynamic general equilibrium model for an economy specialized in tourism to explore long term effects of some environmental and tourism policies. Specifically, we compare the long term effects of two alternative policy instruments, that is, an overnight stay tax and a quality standard imposed to the tourism firms. We show that both instruments have different effects on the incentives to accumulate capital. Moreover, from a long term perspective, the quality standard could be a better instrument than the overnight stay tax since the former implies lower taxation on capital. However, under the quality standard, the incentives for capital accumulation could be excessive thereby leading to dynamic inefficiency.

\section{ACKNOWLEDGEMENTS}

We acknowledge Michael McAleer for his very helpful comments.

\section{REFERENCES}

\footnotetext{
${ }^{1}$ This is always true since $\alpha<\alpha /(\alpha+\gamma)<(1-\gamma)$.
} 
Davies, T. and S. Cahill (2000) Environmental Implications of the Tourism Industry, Resources for the Future Discussion Paper 00-14 (2000).

Gómez, C.M., J. Lozano and J. Rey-Maquieira (forthcoming) Environmental policy and long-term welfare in a tourism economy, Spanish Economic Review

Hettich, F. (1998) Growth effects of a revenueneutral environmental tax reform, Journal of Economics 67, 287-316.

Stokey, N. (1998) Are there limits to growth?, International Economic Review 39, 1-31.

Tahvonen, O. and J. Kuuluvainen (1993) Economic growth, pollution and renewable resources, Journal of Environemtal Economics and Management 24, 101-118.

Tisdell C.A. (2001) Tourism Economics, the Environment and Development Edward Elgar, Cheltenham.

\section{APPENDIX: Stability of the steady state}

In this appendix we discuss the steady state stability conditions under the assumptions that the policy instruments are constant and $s_{A}=, s_{B}=$

Combining (10), (11), (12) (2') and (5) we arrive at:

$$
\begin{gathered}
\dot{C}=\frac{C}{\theta}\left\{\left[\phi\left(\frac{1-\alpha-\gamma}{\tau_{T}}\right)^{1-\beta-\gamma}\left(1-\tau_{T R}\right)^{1-\beta-\gamma} \beta^{\beta} \gamma^{\gamma} N^{\mu}\right]^{\prime / \alpha}\left[\frac{\alpha \tau_{T}}{1-\alpha-\gamma}-\gamma(1-\theta) z \frac{K}{N}\right]-\delta-\rho+\gamma(1-\theta)\right) \\
\dot{K}=\left\{\left[\phi\left[\frac{\left(1-\tau_{T R}\right)(1-\alpha-\gamma)}{\tau_{T}}\right]^{1-\alpha-\beta-\gamma} \beta^{\beta} \gamma^{\gamma} N^{\mu}\right]^{1 / \alpha}(1-\beta-\gamma)-\delta\right\} K-C \\
\dot{N}=\varsigma(\bar{N}-N)-z\left[\phi\left[\frac{\left(1-\tau_{T R}\right)(1-\alpha-\gamma)}{\tau_{T}}\right]^{1-\beta-\gamma} \beta^{\beta} \gamma^{\gamma} N^{\mu}\right]^{1 / \alpha} K
\end{gathered}
$$

Linearization around the steady state results in a system whose Jacobian is:

$$
B=\left(\begin{array}{lll}
b_{11} & b_{12} & b_{13} \\
b_{21} & b_{22} & b_{23} \\
b_{31} & b_{32} & b_{33}
\end{array}\right)
$$

$b_{11}=0$

$b_{12}=-\frac{z v(1-\theta)(1-\alpha-\gamma)(\delta+\rho)}{\theta \tau_{T} \alpha} \frac{C^{*}}{N^{*}}$

$b_{13}=\frac{1}{\theta}\left[\frac{\mu(\delta+\rho)}{\alpha}-v(1-\theta)_{\varsigma}\left(1+\frac{\mu}{\alpha} \frac{\bar{N}-N^{*}}{N^{*}}\right)\right] \frac{C^{*}}{N^{*}}$

$b_{21}=-1$

$b_{22}=\frac{(\delta+\rho)(1-\beta-\gamma)}{\alpha\left(1-\tau_{T R}\right)}-\delta$

$b_{23}=\frac{\mu(1-\beta-\gamma)(\delta+\rho)}{\alpha^{2}\left(1-\tau_{T R}\right)} \frac{K^{*}}{N^{*}}$

$b_{31}=0$

$b_{32}=-z \frac{(1-\alpha-\gamma)(\delta+\rho)}{\alpha \tau_{T}}$

$b_{33}=-\left[\varsigma+\frac{\mu}{\alpha} \frac{\varsigma\left(N-N^{*}\right)}{N^{*}}\right]$

The determinant of $\mathrm{B}$ is:

$$
|B|=z \frac{(1-\alpha-\gamma)(\delta+\rho)^{2} \mu}{\tau_{T} \alpha^{2} \theta} \frac{C^{*}}{N^{*}}>0
$$

The determinant is positive and therefore there are two possibilities: three positive eigenvalues or one ppsitive and two negative. The characteristic equation is:

$$
\hat{\lambda}\left\{\Delta-\varsigma_{-}-\frac{\mu\left(\bar{N}-N^{*}\right)}{\alpha N^{*}}\right\} \mathcal{R}+\left\{\delta \frac{\mu\left(\bar{N}-N^{*}\right)}{\alpha N^{*}}-\phi\right\} \lambda-B=0
$$

where $\Delta=\frac{\delta\left[1-\alpha\left(1-\tau_{T R}\right)-\beta-\gamma\right]+\rho(1-\beta-\gamma)}{\alpha\left(1-\tau_{T R}\right)}$

This equation cannot be solved analytically. However if we set $\theta=\alpha\left(1-\tau_{T R}\right) /(1-\beta-\gamma)>0$ the eigenvalues are:

$$
\lambda_{1}=\Delta
$$




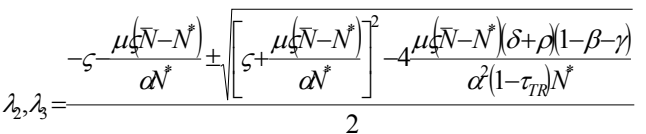

The first eigenvalue is positive since $1-\alpha-\beta-\gamma>0$ and $0 \leq \tau_{T R}<1$. The other two are negative because:

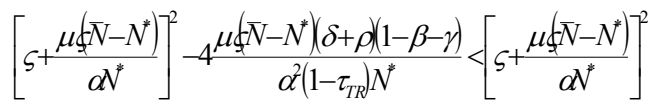

Therefore, provided that the roots are real, the steady state is a saddle-path. Different values for the intertemporal elasticity of substitution mean horizontal shifts of the characteristic equation. Therefore, this result would hold for a large range of values for $\theta$. 